\title{
Composite scaffolds of mesoporous bioactive glass and polyamide for bone repair
}

This article was published in the following Dove Press journal:

International Journal of Nanomedicine

19 May 2012

Number of times this article has been viewed

Jiacan $\mathrm{Su}$

Liehu Cao

Baoqing Yu

Shaojun Song

Xinwei Liu

Zhiwei Wang

Ming Li

Department of Orthopedics, Changhai Hospital, Second Military

Medical University, Shanghai, China
Correspondence: Zhiwei Wang; Ming Li Department of Orthopedics, Changhai Hospital, Second Military Medical

University, 800 Xiangyin Road,

Shanghai 200433, China

Tel +862181873400

Fax +862181873398

Email sujiacan2012@yahoo.cn; nic750@263.net
Abstract: A bone-implanted porous scaffold of mesoporous bioglass/polyamide composite (m-BPC) was fabricated, and its biological properties were investigated. The results indicate that the m-BPC scaffold contained open and interconnected macropores ranging 400-500 $\mu \mathrm{m}$, and exhibited a porosity of $76 \%$. The attachment ratio of MG-63 cells on m-BPC was higher than polyamide scaffolds at 4 hours, and the cells with normal phenotype extended well when cultured with m-BPC and polyamide scaffolds. When the m-BPC scaffolds were implanted into bone defects of rabbit thighbone, histological evaluation confirmed that the m-BPC scaffolds exhibited excellent biocompatibility and osteoconductivity, and more effective osteogenesis than the polyamide scaffolds in vivo. The results indicate that the m-BPC scaffolds improved the efficiency of new bone regeneration and, thus, have clinical potential for bone repair.

Keywords: mesoporous bioglass, polyamide, composite scaffolds, biocompatibility, bone repair

\section{Introduction}

Bioactive glasses (BGs) with excellent biocompatibility and bioactivity have been used for bone tissue repair since their discovery some decades ago. ${ }^{1,2}$ In 2004, a significant evolution in this field was developed by Yan et al who synthesized, for the first time, BG that showed an ordered mesoporous arrangement, and the inclusion of mesopores in silicon dioxide-calcium oxide-phosphorus pentoxide-based glasses opened a wide range of new potential applications for $\mathrm{BG} .^{3}$

Mesoporous BG (m-BG) shows the outstanding textural properties of classical silica-based mesoporous materials such as MCM-41 and SBA-15, that is, high surface area and pore volume, and well-defined mesoporous diameter., ${ }^{4,5}$ The large surface area of $\mathrm{m}-\mathrm{BG}$ results in higher chemical reactivity compared to that of BG. The existence of mesoporosities in the BG matrixes and their high surface area greatly accelerate the deposition process of hydroxycarbonate apatite as demonstrated by Zhao et al. ${ }^{6}$ Thus, bioactive kinetics are enhanced, which improves the role of silica-based BG as devices for bone tissue regeneration. In this sense, several groups have proposed $\mathrm{m}-\mathrm{BG}$ as components of scaffolds for bone tissue engineering. ${ }^{7-9}$ Although the existing bioactive inorganic materials (such as BG) possess excellent bioactivity, they are very brittle and have inherently poor tensile properties. ${ }^{10,11}$ Polymers such as poly(lactic acid), polyglycolide, and poly(lactide-co-glycolide) have been widely used to fabricate different types of scaffolds for bone repair, owing to their biocompatibility and malleable nature. ${ }^{12}$ Nevertheless, there are limits to the practical use of these polymers for bone replacement. For example, none of the polymers mentioned above are bioactive, 
which means that the newly formed bone tissue cannot bond tightly to the polymer surface. ${ }^{13}$ Given the limitations and advantages of bioactive inorganic materials and polymers, the application of polymers and bioactive materials to create a bioactive composite scaffold was suggested, and many studies have demonstrated composite scaffolds with physical, biological, and mechanical properties suitable for bone tissue engineering application. ${ }^{14-16}$

Tissue engineering offers a promising new approach to bone repair. Successful bone tissue engineering requires the use of a porous scaffold with interconnected spaces in order to provide sufficient room for cell migration and adhesion, and the ingrowth of new bone tissue. ${ }^{17,18}$ Polyamide (PA), a polymer with excellent biocompatibility, has been used to fill bone defects and to create porous scaffolds for bone tissue engineering. ${ }^{19}$ Poly(lactic acid), or polyglycolide and poly(lactide-co-glycolide), show a collapse degradation manner after implanted in vivo, and also give rise to acid degradation by-products, stimulation to tissue, and even inflammation, while PA does not have these disadvantages. ${ }^{12}$ In this study, a novel porous scaffold of $\mathrm{m}-\mathrm{BG}$ and PA6 composite (m-BPC) was fabricated, and its properties were investigated.

\section{Material and methods Preparation of $m-B G$}

$\mathrm{m}-\mathrm{BG}$ was synthesized by using nonionic block copolymer $\mathrm{EO}_{20} \mathrm{PO}_{70} \mathrm{EO}_{20}$ (Pluronic P123, Boston, USA) (Sigmaaldrich, Shanghai Trading Co., Ltd, Shanghai, China) as the template agent, ${ }^{4}$ and tetraethyl orthosilicate (Sinopharm Chemical Reagent Co., Ltd, Shanghai, China) as the silica source. P123 (8 g) was dissolved in $120 \mathrm{~mL}$ ethanol under stirring for 2 hours, and $13.4 \mathrm{~g}$ of tetraethyl orthosilicate and $4 \mathrm{~g}$ of $1 \mathrm{M}$ hydrochloride (Sinopharm Chemical Reagent Co., Ltd, Shanghai, China) were added to the mixed solution of P123 and ethanol. Then, $2.8 \mathrm{~g}$ of calcium nitrate tetrahydrate (Sinopharm Chemical Reagent Co., Ltd, Shanghai, China) and $1.46 \mathrm{~g}$ of triethyl phosphate (Sinopharm Chemical Reagent Co., Ltd, Shanghai, China), with a molar ratio of 1:1.5 calcium:phosphorus, were added as calcium and phosphorus oxide precursors, respectively, and stirred magnetically at room temperature for 24 hours. The precipitation was filtered, washed thoroughly with deionized water, and dried at $60^{\circ} \mathrm{C}$ under vacuum (DIF6020, Shanghai Jinghong laboratory equipment Co., Ltd, Shanghai, China) to get the powders. The samples were sintered at $600^{\circ} \mathrm{C}$ for 6 hours with a heating rate of $1^{\circ} \mathrm{C} /$ min to remove the template $(\mathrm{P} 123)$ and obtain $\mathrm{m}-\mathrm{BG}$. Scanning electron microscopy (SEM) (JSM-6360 LV;
JEOL Ltd, Tokyo, Japan) and high resolution transmission electron microscopy (TEM) (JEM-2010; JEOL) was used to characterize the morphology and microstructure of m-BG. Brunauer-Emmett-Teller and Barrett-Joyner-Halenda analyses with a porosimeter ( $\operatorname{TriStar}^{\circledR} 3000$; Micromeritics Instrument Corporation, Norcross, GA) were used to determine the surface area and the pore size distribution.

\section{Preparation of $m-B P C$ scaffolds}

The m-BPC scaffolds were prepared by solvent casting and particulate leaching. Briefly, $9 \mathrm{~g}$ of PA6 (Bayer Durethan ${ }^{\circledR}$ B30S, Leverkusen, Germany) was dissolved in $50 \mathrm{~mL}$ calcium chloride/ethanol solution at a concentration of $20 \%$ (weight/ volume), and $3 \mathrm{~g} \mathrm{~m}-\mathrm{BG}$ was added to produce an m-BG/PA composite with 25 weight percent $\mathrm{m}-\mathrm{BG}$ content. The mixture was stirred continuously for 2 hours, and sodium chloride was added as a porogen (size: 400-500 $\mu \mathrm{m}$ : PA/sodium chloride $=1 / 8$, weight $/$ weight , and the mixture was cast into Teflon molds containing 60 wells (Institute of biomaterials, Shanghai, China) $(\Phi 10 \times 5 \mathrm{~mm})$. The samples were air-dried in a fume hood (Institute of Biomaterials, Shanghai, China) for 24 hours to evaporate the ethanol and were subsequently vacuum-dried at $50^{\circ} \mathrm{C}$ for 48 hours to remove any remaining solvent. To leach out the salt, the dry samples were immersed in deionized water for 48 hours at room temperature, with water changes approximately every 12 hours three or four times. The scaffolds were obtained by removing from the water and air-drying for 24 hours. Using the same method, pure PA scaffolds were prepared as a control. The morphology of m-BPC scaffolds were observed by SEM (JSM-6360LV; JEOL). The porosity of the m-BPC scaffolds was measured in distilled water using Archimedes method.

\section{Cell attachment}

To investigate the attachment properties of MG-63 cells (Chinese Academy of Sciences Shanghai cell library, Shanghai, China) on the m-BPC scaffolds (PA as a control), the samples $(\Phi 10 \times 5 \mathrm{~mm})$ were sterilized using ultraviolet light. MG-63 cells were seeded on the samples at a density of $5 \times 10^{3}$ cells/per sample. Adhesion cells on substrates were assessed quantitatively using a 3-(4,5- dimethylthiazol-2-yl)2,5-diphenyltetrazolium bromide (MTT) assay (MTT Kit; Roche Diagnostics, Indianapolis, IN). In brief, cells/scaffold construct was placed in a culture medium containing MTT and incubated in a humidified atmosphere at $37^{\circ} \mathrm{C}$ for 4 hours. The absorbance value was measured at $570 \mathrm{~nm}$ using a Multiskan MK3 microplate reader (Thermoelectric, Shanghai, China). Six specimens were tested at each incubation period, and 
each test was performed in triplicate. Results are reported as optical density units. The morphologies of cells cultured with both m-BPC and PA scaffold samples were observed and photographed under an inverted light microscope (IMT-2, A10PL; Olympus Corporation, Tokyo, Japan).

\section{Surgical procedures}

The study was approved by the Laboratory Animal Center of Shanghai University of Traditional Chinese Medicine (Shanghai, China). A study in a rabbit femur cavity defect model was carried out to investigate the efficacy of composite scaffolds in promoting bone repair. Twentyfour skeletal mature New Zealand white rabbits (Silaike Inc. Shanghai, China) were used in this study, and surgical intervention was performed under general anesthesia. Lateral and medial approaches were performed in left shaved knees to expose the distal femoral epiphysis, and the cavitary defects were created with a medium speed burr $(\Phi 5 \times 5 \mathrm{~mm})$. After the defects were washed with physiological saline, the $\mathrm{m}-\mathrm{BPC}$ and PA scaffolds were implanted; the empty bony defects served as the control. At 4 weeks and 12 weeks after implantation, the rabbits were sacrificed with an overdose of pentobarbital and the bone formation was evaluated with synchrotron radiationbased microcomputed tomography (SR m-CT) BL13W1, Shanghai Synchrotron Radiation Facility. Shanghai, China scan images. The implants were then retrieved with the surrounding tissues and prepared for histological analysis.

\section{SR m-CT imaging and histological analysis}

SR m-CT was used to evaluate repair of the bone defect by composite scaffolds, which were performed at beamline BL13W (Shanghai Synchrotron Radiation Facility, Shanghai,
China) using a monochromatic beam with an energy of $30 \mathrm{keV}$ and a sample-to-detector distance of $1.5 \mathrm{~m}$. A $4000 \times 2500$ charge-coupled device detector, (CCD, Double Asahi Electronics Co., Ltd, Shanghai, China), with the pixel size set to $6 \mathrm{~mm}$, was used to record images. A total of 1200 projections within an angular range of $180^{\circ} \mathrm{C}$ were taken, and the exposure time amounted to 8 seconds per projection. The three-dimensional structure was reconstructed using a filtered back-projection algorithm. The images were finally redigitized with an 8-bit data format, proportional to the measured attenuation coefficients of the voxels. Bone radiomorphometric analysis was performed by using MicroView 2.2 Advanced Bone Analysis Application software (GE Healthcare, Waukesha, WI). The amount of bone ingrowth into the scaffolds was quantified as the bone volume within the defined volume of interest in each bone defect site.

At the end of each implantation period, femora were removed and assigned to histological analysis. After fixation with $4 \%$ neutral buffered formalin for 48 hours, the extracted femora were decalcified in $12.5 \%$ ethylenediaminetetraacetic acid (Sinopharm Chemical Reagent Co., Ltd, Shanghai, China), dehydrated in a graded series of alcohol, and embedded in paraffin. Serial 4-mm thick sections were then stained with hematoxylin and eosin (The Tianjin Bankee Biotechnology Co., Ltd,Tianjin,China) and observed microscopically.

\section{Statistical analysis}

All quantitative data were analyzed with Origin ${ }^{\circledR} 8.0$ (OriginLab Corporation, Northampton, MA) and expressed as mean and standard deviation. Statistical analysis was carried out using analysis of variance. Statistical significance was attained with greater than $95 \%$ confidence level $(P<0.05)$.
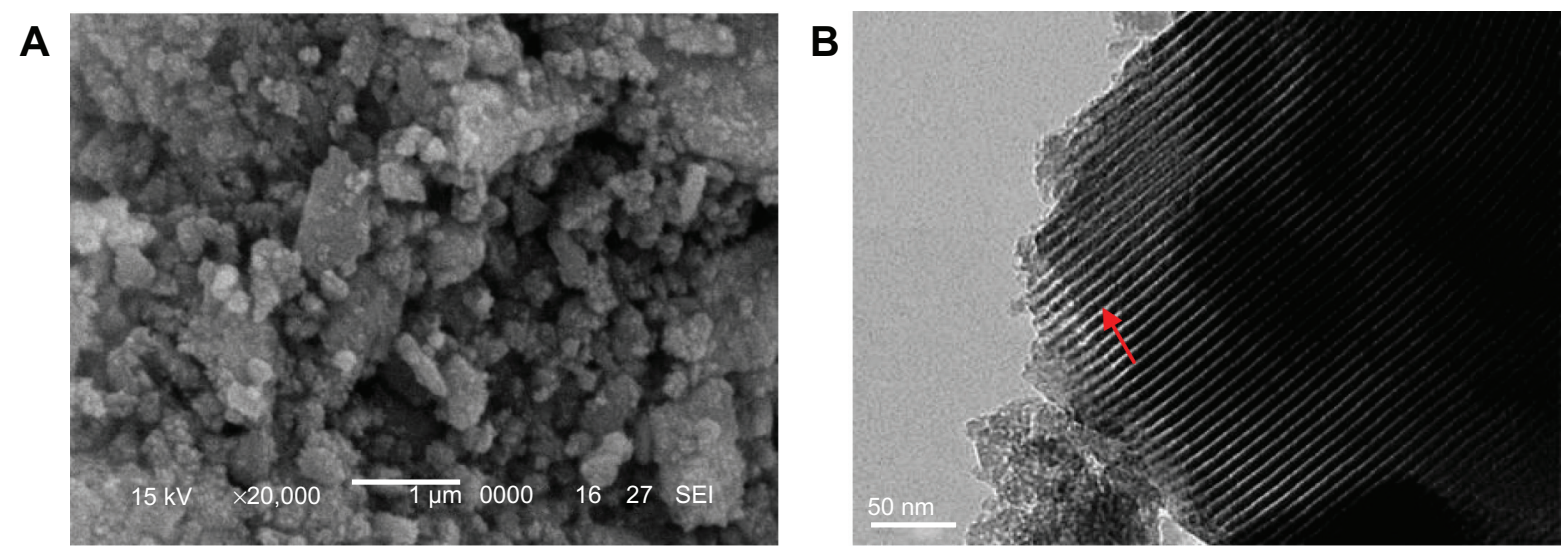

Figure I (A) Scanning electron microscopic and (B) transmission electron microscopic images of the morphology of mesoporous bioactive glass. Note: Arrow represents mesoporous channels. 
A

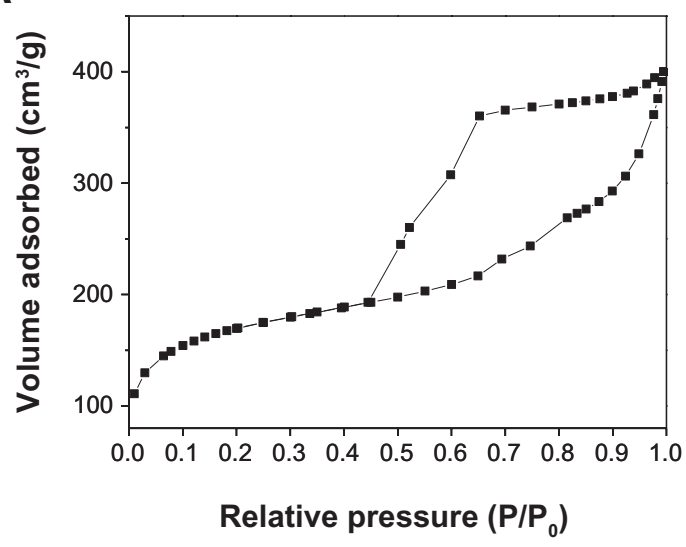

B

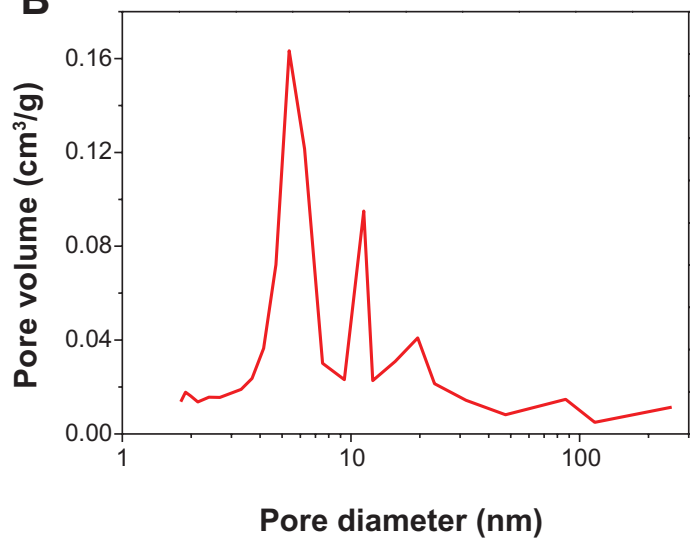

Figure 2 (A) Nitrogen gas sorption isotherms and (B) pore size distribution of mesoporous bioactive glass.

\section{Results}

\section{SEM and TEM analysis}

SEM and TEM images of the $\mathrm{m}-\mathrm{BG}$ are shown in Figure 1. Approximate sphere-like m-BG particles with a size of $0.1-1 \mu \mathrm{m}$ are shown as an SEM image in Figure 1A. In the TEM image, it was found that homogeneously distributed mesopore channels appeared in the BG particle (shown as white line; Figure 1B).

\section{Brunauer-Emmett-Teller analysis}

The sorption isotherms and pore size distribution of $\mathrm{m}-\mathrm{BG}$ are shown in Figure 2. The results show that the specific surface area and average pore size of m-BG were $439 \mathrm{~m}^{2} \mathrm{~g}^{-1}$ and $7 \mathrm{~nm}$, respectively. The nitrogen adsorption-desorption isotherm of the m-BG exhibited type IV adsorption behavior, which indicates capillary condensation in mesopores. The results indicate that the prepared $\mathrm{m}-\mathrm{BG}$ had a mesoporous structure.

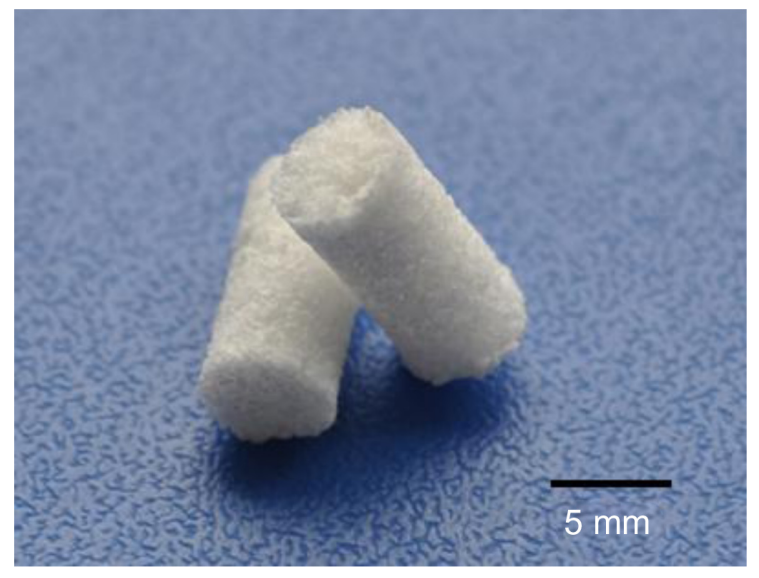

Figure 3 Photo of mesoporous bioactive glass and polyamide composite scaffolds.

\section{Morphology and microstructure of $m$-BPC scaffolds}

Figure 3 shows a photo of m-BPC scaffolds. Figure 4 shows the SEM images of the surface morphology and microstructure of the $\mathrm{m}$-BPC porous scaffolds under various magnifications. The m-BPC scaffolds exhibited a macroporous structure with completely open interconnected pores. The pores appeared almost spherical in shape, with diameters of $400-500 \mu \mathrm{m}$ (Figure 4A). High-magnification SEM images further revealed that a number of small pores (around $2 \mu \mathrm{m}$ ) were distributed across the macropore walls. The porosity of the m-BPC scaffolds prepared by this method was around $76 \%$.

\section{Cell attachment and morphology}

Cell attachment was investigated using the MTT assay of MG-63 cells cultured on m-BPC scaffold samples; PA scaffolds and tissue culture plate were used as controls. Figure 5 shows the results of the optical density values (represents cell attachment ratio) for these scaffold samples. At 4 hours, the optical density values for $\mathrm{m}$-BPC were significantly higher than PA and the control $(P<0.05)$. These results indicate that cell attachment for m-BPC was superior to PA samples, suggesting that $\mathrm{m}-\mathrm{BPC}$ facilitated cell adhesion on its surfaces.

Morphologies of MG-63 cells cultured with m-BPC and PA scaffold samples were observed using phase contrast microscopy, as shown in Figure 6. The micrographs reveal that after being cultured for 4 hours, MG-63 cells cultured with both m-BPC and PA specimens grew well and stretched sufficiently, had full configurations, and showed no abnormal morphologies. These results indicate that both $\mathrm{m}-\mathrm{BPC}$ and PA scaffolds had no negative effect on MG-63 cell morphology and viability, and both provide good cytocompatibility. 

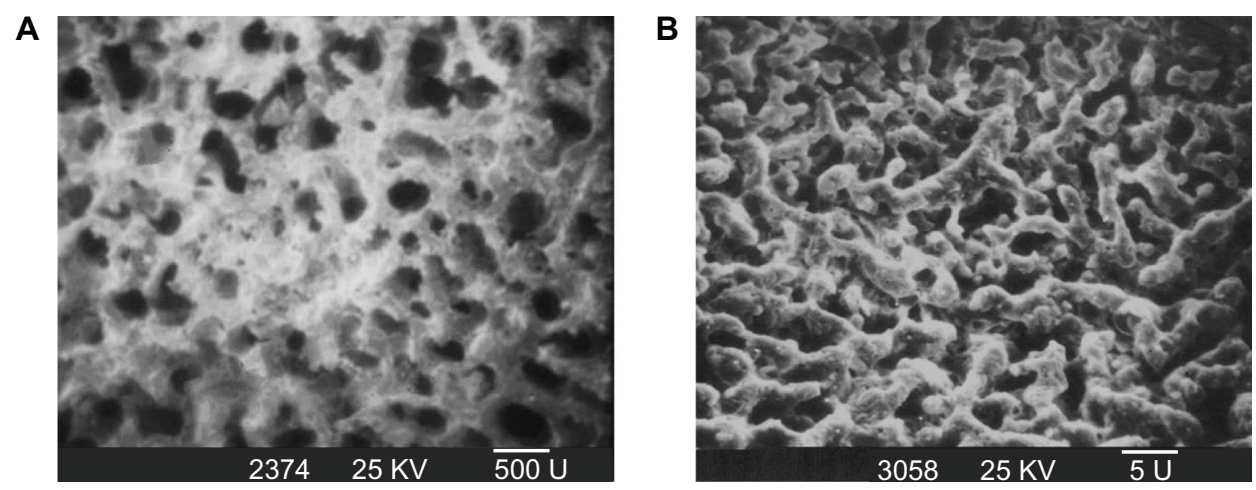

Figure 4 Scanning electron microscopic photographs of mesoporous bioactive glass and polyamide composite scaffolds at $(\mathbf{A}) \times 50$ and $(\mathbf{B}) \times 5000$ magnification

\section{Implantation of $\mathrm{m}$-BPC scaffolds in vivo Macroscopic and SR m-CT evaluation}

Figure 7 shows the macroscopic evaluation of the m-BPC scaffolds implanted in the bone cavities of rabbit femora for 4 weeks and 12 weeks. At 4 weeks, abscission of the suture occurred and the surface of the bone defects was partially filled with callus bone. At 12 weeks, the bone defects were repaired by implanted scaffolds; the wounds healed well without dehiscence and did not elicit any obvious inflammatory response in the adjacent soft tissue. No signs of implant rejection, necrosis, or infection were found at the experimental time.

The m-BPC scaffolds implanted into bone defects of rabbit femora were scanned by SR m-CT at 4 weeks and 12 weeks to evaluate the in vivo bone ingrowth of $\mathrm{m}$-BPC scaffolds (Figure 8). At 4 weeks, the three-dimensional SR m-CT images revealed that only a small amount of newly formed bone appeared in the m-BPC scaffolds at the native bone margins and the defect periphery throughout the cross-

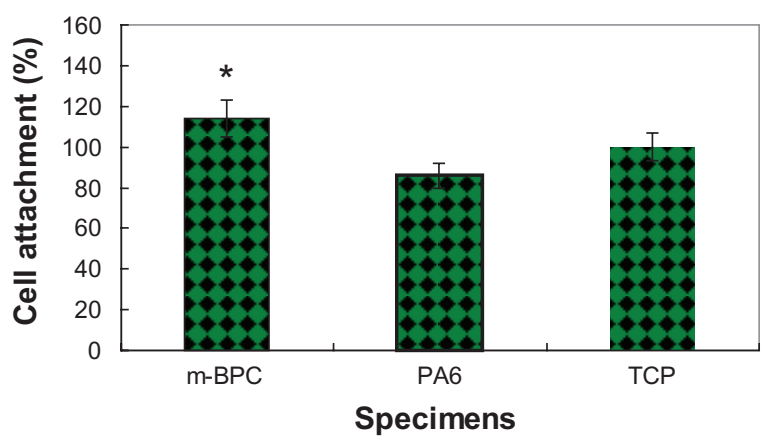

Figure 5 Attachment of MG-63 cells on mesoporous bioactive glass and polyamide composite scaffolds. Polyamide scaffolds and tissue culture plate were used as controls. Cell attachment is compared to the tissue culture plate control (100\%).

Notes: *Statistical analysis: cell attachment ratio for $\mathrm{m}-\mathrm{BPC}$ were significantly higher than PA and the control $(P<0.05)$

Abbreviations: $\mathrm{m}-\mathrm{BPC}$, mesoporous bioactive glass and polyamide composite; PA6, polyamide 6; TCP, tissue culture plate. section of the bone defect. At 12 weeks, the most extensive bone ingrowth was observed throughout the entire volume of the m-BPC scaffolds, and the formation of new bone tissue was observed as well.

\section{Histological analysis}

Histological analysis of the m-BPC scaffolds implanted into bone defects of rabbit femora, as shown in Figure 9, enabled a more detailed analysis on the new bone tissue formation inside the m-BPC scaffolds. At 4 weeks, new bone formation was found around the scaffold materials in the vicinity of bone marrow. This newly formed bone had a more trabecular appearance with osteoid depositions at the surface of the scaffold materials (Figure 9A). After 12 weeks, newly formed bone was predominant in the healing area and woven bone was replaced by mature trabecular bone. New bone formation (bone ingrowth) in the m-BPC scaffolds was more extensive (Figure 9B), which was consistent with SR $\mathrm{m}-\mathrm{CT}$ analysis.

Figure 10 shows the regenerated bone volume within the bone defect, which was used to evaluate the repair effects of bone defects after m-BPC and PA scaffolds implanted for 4 weeks and 12 weeks. The results indicate that a significant increase in bone volume was observed for both m-BPC and PA scaffolds from 4 weeks to 12 weeks. In addition, the $\mathrm{m}$-BPC scaffolds contained higher bone volume than the PA scaffolds (control) at both 4 weeks and 12 weeks $(P<0.05)$.

\section{Discussion}

Recently, supramolecular chemistry has allowed the emergence of a new generation of advanced mesoporous biomaterials with high surface area, high porosity, and uniform pore channels, which show enhanced bioactivity and have potential for use in the fabrication of porous scaffolds for bone regeneration. ${ }^{20,21} \mathrm{In}$ 

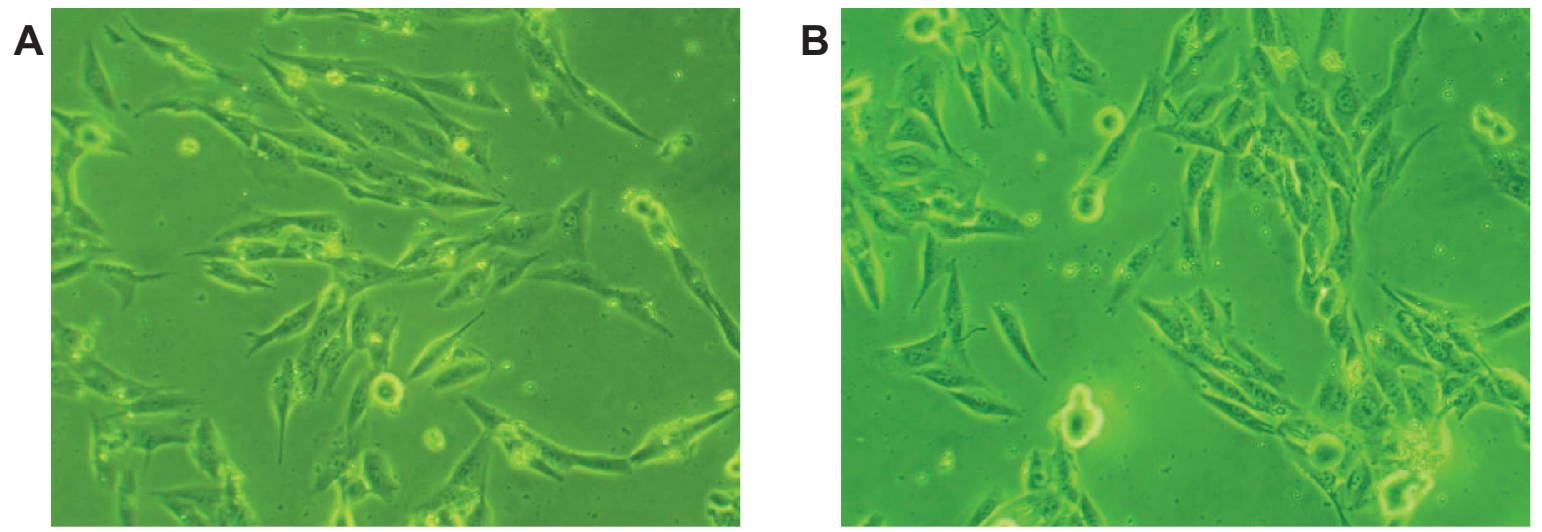

Figure 6 Phase contrast microscopic photographs of MG-63 cells cultured with (A) mesoporous bioactive glass and polyamide composite scaffolds and (B) and polyamide scaffolds for 4 hours.

this study, m-BG were fabricated by using the sol-gel method and P123 as a template agent. The surface area and average pore size of the $\mathrm{m}-\mathrm{BG}$ were $439 \mathrm{~m}^{2} \mathrm{~g}^{-1}$ and $7 \mathrm{~nm}$, respectively. Previous studies have shown that increasing the surface area of the biomaterials might greatly accelerate the kinetic process of apatite deposition and, therefore, enhance bone-forming bioactivity. ${ }^{22}$

There have been a number of studies in the literature focusing on the composites created by combining polymers and bioactive inorganic biomaterials. ${ }^{23-25}$ To the best of the authors' knowledge, there was no previous report about the preparation of m-BPC scaffolds used for bone repair material. It is expected that if the synthesis of $\mathrm{m}-\mathrm{BG}$ has a high surface area, the bioactivity of the composite scaffolds should be improved. Therefore, the m-BPC scaffolds were prepared, and its biological properties were investigated in the current study. An ideal scaffold for bone repair should possess appropriate architecture and properties to provide a biological environment for cell attachment and cell/tissue ingrowth. ${ }^{26,27}$ The m-BPC scaffolds, containing 25 weight percent $\mathrm{m}$-BG content with $76 \%$ porosity, exhibited interconnected porous network and large pore sizes $(400-500 \mu \mathrm{m})$, which might meet the demand of the porous structure. The mesoporous material of $\mathrm{m}-\mathrm{BG}$ in the composite might greatly increase the surface area of scaffold materials, which is favorable for cell extension and, thus, facilitates repopulating the entire bone defect with cells.

Cell attachment belongs to the first phase of cell/material interactions, which influences cell growth and morphology and proliferates upon contact with the biomaterials. ${ }^{28} \mathrm{In}$ the current study, an MTT assay was used to assess the relative number of cells that adhered to the m-BPC scaffold materials because optical density absorbance values can be used as indicators of the relative number of cells attached on substrate materials. The results show that the MG-63 cells adhered better to the m-BPC scaffolds than the PA scaffolds
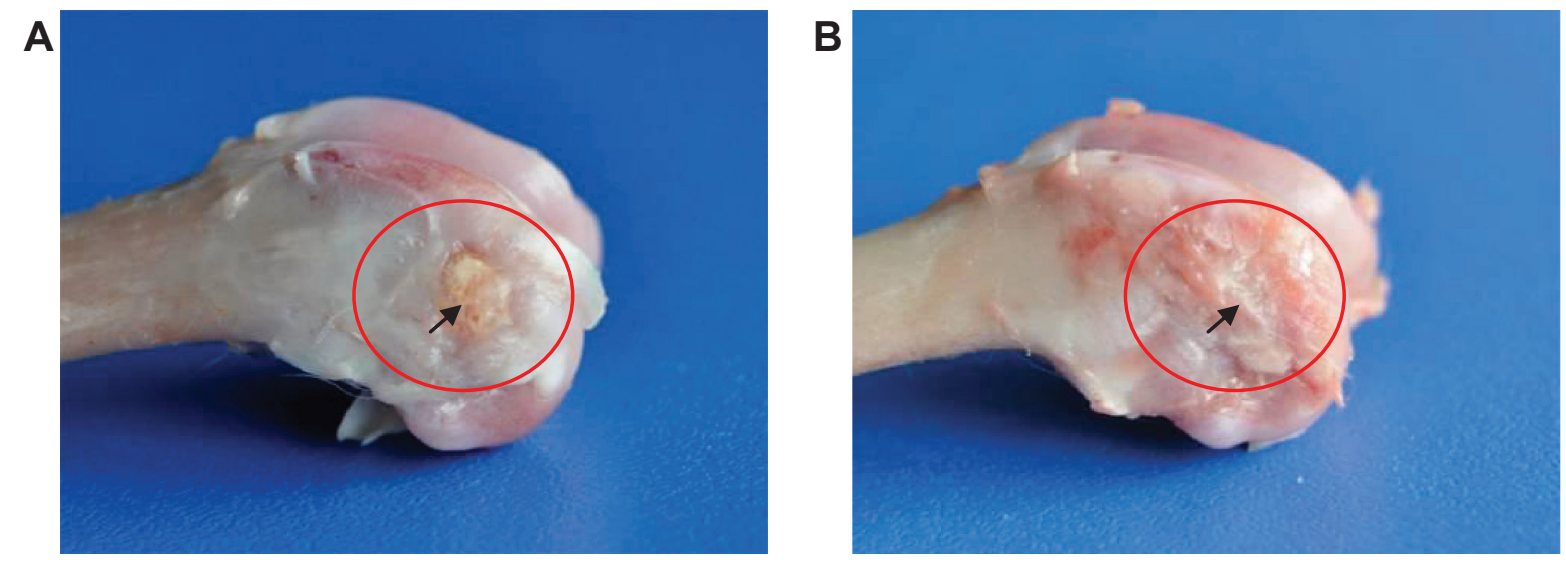

Figure 7 Macroscopic evaluation of mesoporous bioactive glass and polyamide composite scaffolds implanted into bone defects of rabbit femora for (A) 4 weeks and (B) 12 weeks.

Note: Circle and arrow show the bone defect area site. 

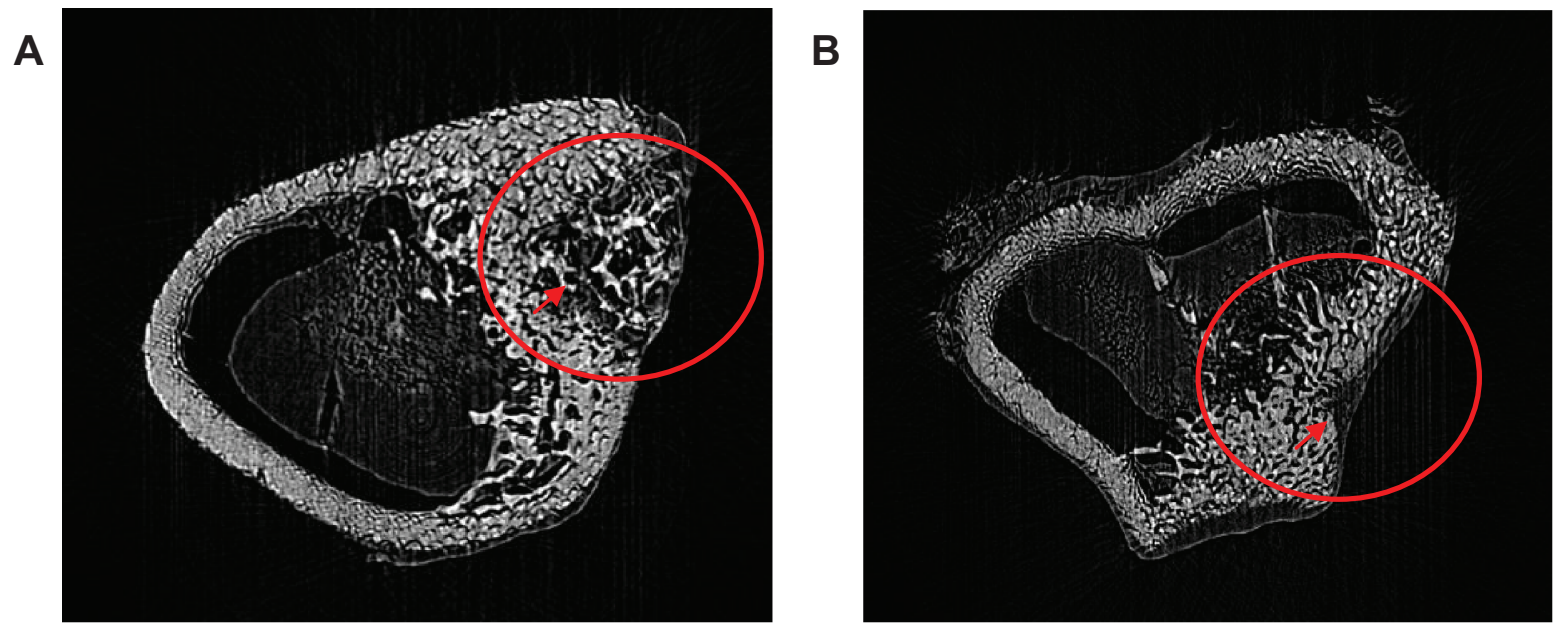

Figure 8 Synchrotron radiation-based microcomputed tomography of a three-dimensional reconstruction of cross-section images of mesoporous bioactive glass and polyamide composite scaffolds implanted into bone defects of rabbit femora for (A) 4 weeks and (B) 12 weeks. Note: Circle and arrow show the bone defect area site.

A

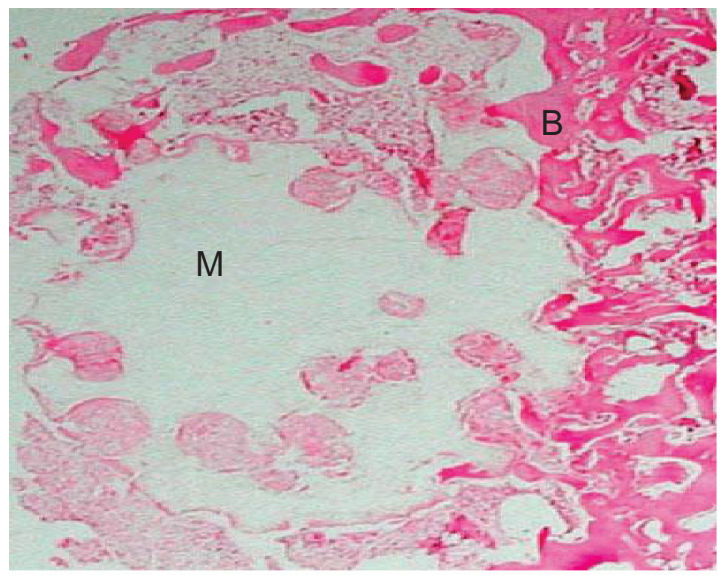

C

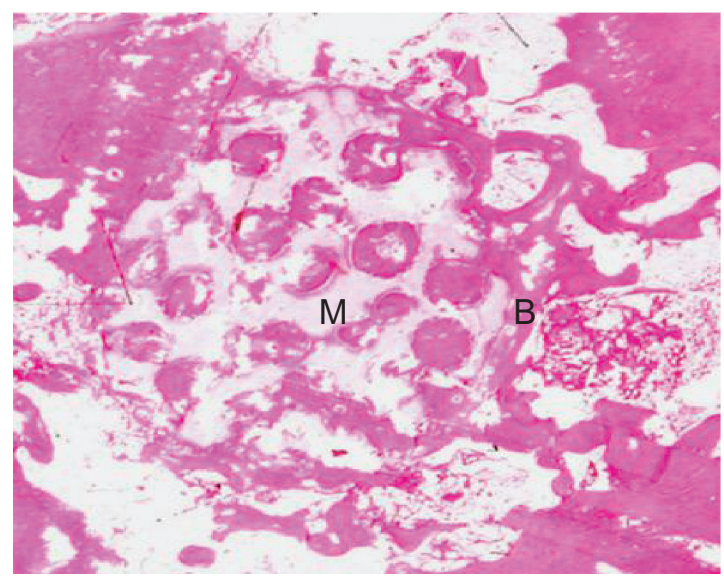

B

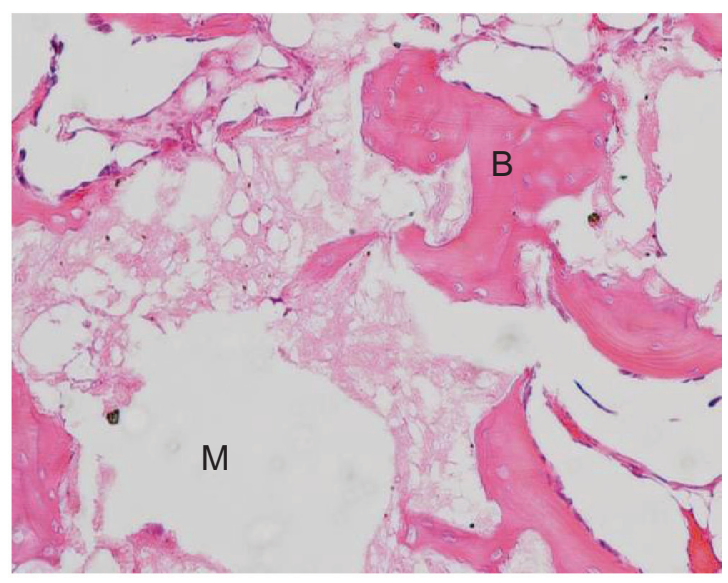

D

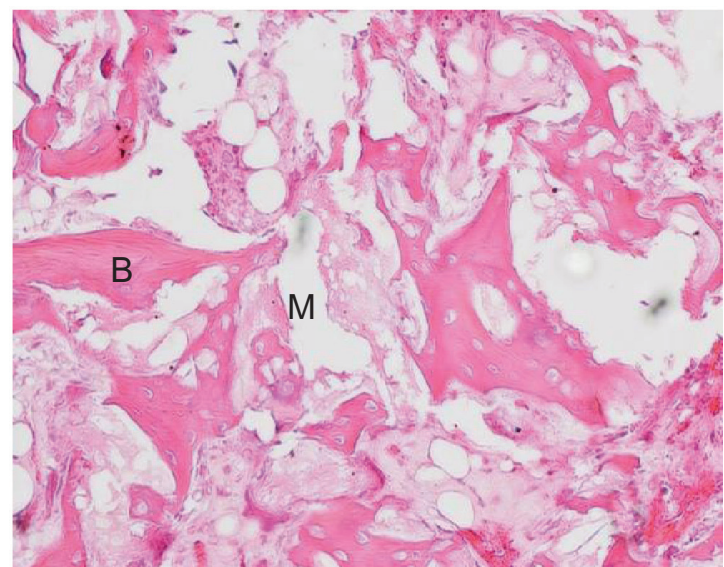

Figure 9 Hematoxylin and eosin stained section of mesoporous bioactive glass and polyamide composite scaffolds implanted into bone defects of rabbit femora for (A and $\mathbf{B})$ 4 weeks ( $\times 5$ and $\times 20$, respectively) and (C and D) 12 weeks $(\times 5$ and $\times 20$, respectively).

Notes: $B$ represents the new bone tissue, $M$ represents the biomaterials. 


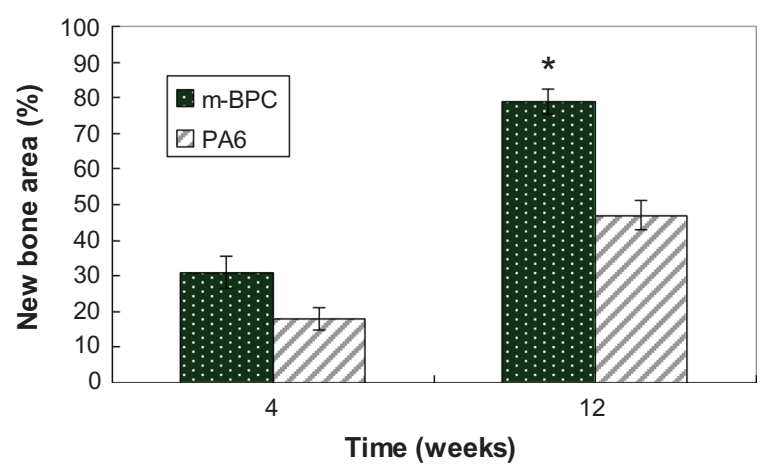

Figure 10 Quantitative analysis of the bone defect area replaced by new bone tissue after mesoporous bioactive glass and polyamide composite scaffolds, and polyamide scaffolds were implanted in vivo for 4 weeks and 12 weeks.

Notes: *Statistical analysis: new bone area ratio for $\mathrm{m}$-BPC were significantly higher than PA6 $(P<0.05)$.

Abbreviations: m-BPC, mesoporous bioactive glass and polyamide composite; PA6, polyamide 6 .

and tissue culture plate within the first 4 hours of culture. The enhancement of MG-63 attachment on m-BPC is likely associated with its material surface features, such as pore size, surface adsorption ability, and protein adsorption of the scaffolds in cell culture medium. The m-BPC may have special surface properties, due to the addition of $\mathrm{m}-\mathrm{BG}$ into PA, that promote cell attachment.

The in vivo biocompatibility of the m-BPC scaffolds was determined by implantation of the scaffold materials into bone defects of rabbit femora. SR m-CT imaging results show the interactions between the m-BPC scaffolds and the surrounding tissues at 4 weeks and 12 weeks; the bone regeneration process began soon after implantation, indicating that the scaffolds created an environment more conducive for bone regeneration.

Another structural property favorable to the osteogenic capacity of m-BPC scaffolds may be related to their mesoporous texture. The mesoporous structure provides a larger specific area and faster dissolution rate compared to traditional melt-derived BGs. ${ }^{29}$ As most of the mammalian cells are anchorage dependent, the mesoporous matrix also facilitates peptide adsorption onto the external surface of m-BPC scaffolds after implantation, making it more accessible to the osteoblastic cell microenvironment that provides a protein-rich surface for cell attachment, differentiation, and migration to form new tissues. ${ }^{30}$ The morphology of the interface between the m-BPC scaffolds and host bone tissue after implantation for 4 weeks and 12 weeks was observed by histological evaluation. The results show that new bone tissue was found to extend along the m-BPC scaffolds' surface and grow into the pores of the scaffolds. The results demonstrate that the m-BPC scaffolds had excellent biocompatibility and osteoconductivity, and could repair the bone defects.

As the implantation time prolonged, new bone tissue regenerated and gradually penetrated into the m-BPC scaffolds. The area of newly formed bone gradually increased with time: $31 \%$ and $79 \%$ of the bone defect area were separately filled with newly formed bone tissue after m-BPC scaffold implantation at 4 weeks and 12 weeks, respectively, indicating that the hierarchically mesoporous-macroporous structure with large surface area could promote m-BPC osteogenesis. However, the area of newly formed bone was $18 \%$ and $47 \%$ after PA scaffold implantation at 4 weeks and 12 weeks, respectively. The results demonstrate that the m-BPC scaffolds exhibited high efficiency of bone regeneration. Clearly, the m-BPC had good bioactivity by incorporation of m-BG into PA compared to PA scaffolds. In short, the characteristics of m-BPC scaffolds enhanced their bioperformance, and they showed good biocompatibility as well as faster and more effective osteogenesis than PA scaffolds.

\section{Conclusion}

The m-BPC scaffolds, shown to have $76 \%$ porosity and macroporous structure with large $(400-500 \mu \mathrm{m})$ open interconnecting pores, were fabricated for the current study. The results show that the incorporation of $\mathrm{m}-\mathrm{BG}$ into PA to form m-BPC scaffolds improved the cell attachment ratio compared to PA scaffolds. By using SR m-CT imaging and histological analysis, it was found that new bone tissue regenerated and gradually penetrated into the m-BPC scaffolds, and $79 \%$ of the bone defect area was filled with newly formed bone tissue 12 weeks after m-BPC implantation. The results indicate that the m-BPC scaffolds significantly promoted repair of the bone defect in the rabbit femur model and exhibited high efficiency of bone regeneration. In short, the m-BPC scaffolds present not only good biocompatibility, but also faster and more effective osteogenesis than PA scaffolds.

\section{Acknowledgment}

This study was supported by grants from the Major Program of Natural Science Foundation of Shanghai, China (No 11JC1416302).

\section{Disclosure}

The authors report no conflicts of interest in this work.

\section{References}

1. Hench LL, Splinter RJ, Allen WC, Greenlee TK. Bonding mechanisms at the interface of ceramic prosthetic materials. J Biomed Mater Res. 1971;5(6):117-141. 
2. Ducheyne P. Bioceramics: material characteristics versus in vivo behavior. J Biomed Mater Res. 1987;21(Suppl A2):219-236.

3. Yan X, Yu C, Zhou X, Tang J, Zhao D. Highly ordered mesoporous bioactive glasses with superior in vitro bone-forming bioactivities. Angew Chem Int Ed Engl. 2004;43(44):5980-5984.

4. Yan X, Deng H, Huang X, et al. Mesoporous bioactive glasses I. Synthesis and structural characterization. J Non Cryst Solids. 2005; 351(40-42):3209-3217.

5. Yan X, Huang X, Yu C, et al. The in-vitro bioactivity of mesoporous bioactive glasses. Biomaterials. 2006;27(18):3396-3403.

6. Zhao D, Yang P, Chmelka BF, Stucky GD. Multiphase assembly of mesoporous-macroporous membranes. Chem Mater. 1999;11(5): $1174-1178$.

7. Shi QH, Wang JF, Zhang JP, Fan J, Stucky GD. Rapid-setting, mesoporous, bioactive glass cements that induce accelerated in vitro apatite formation. Adv Mater. 2006;18(8):1038-1042.

8. Wu C, Ramaswamy Y, Zhu Y, et al. The effect of mesoporous bioactive glass on the physiochemical, biological and drug-release properties of poly(DL-lactide-co-glycolide) films. Biomaterials. 2009;30(12): 2199-2208.

9. Wang X, Li X, Ito A, Sogo Y. Synthesis and characterization of hierarchically macroporous and mesoporous $\mathrm{CaO}-\mathrm{MO}-\mathrm{SiO}_{2}-\mathrm{P}_{2} \mathrm{O}_{5}$ $(\mathrm{M}=\mathrm{Mg}, \mathrm{Zn}, \mathrm{Sr})$ bioactive glass scaffolds. Acta Biomater. 2011;7(10): 3638-3644.

10. Li X, Shi J, Dong X, Zhang L, Zeng H. A mesoporous bioactive glass/ polycaprolactone composite scaffold and its bioactivity behavior. J Biomed Mater Res A. 2008;84(1):84-91.

11. Wang X, Li X, Onuma K. Mesoporous bioactive glass coatings on stainless steel for enhanced cell activity, cytoskeletal organization and AsMg immobilization. J Mater Chem. 2010;20(31):6437-6445.

12. Kim SS, Utsunomiya H, Koski JA, et al. Survival and function of hepatocytes on a novel three-dimensional synthetic biodegradable polymer scaffold with an intrinsic network of channels. Ann Surg. 1998; 228(1):8-13.

13. Whang K, Goldstick TK, Healy KE. A biodegradable polymer scaffold for delivery of osteotropic factors. Biomaterials. 2000;21(24): 2545-2551.

14. Wu C, Zhang Y, Zhu Y, Friis T, Xiao Y. Structure-property relationships of silk-modified mesoporous bioglass scaffolds. Biomaterials. 2010; 31(13):3429-3438

15. Kang SW, Yang HS, Seo SW, Han DK, Kim BS. Apatite-coated poly(lactic-co-glycolic acid) microspheres as an injectable scaffold for bone tissue engineering. $J$ Biomed Mater Res A. 2008;85(3): $747-756$

16. Kim HW, Knowles JC, Kim HE. Hydroxyapatite porous scaffold engineered with biological polymer hybrid coating for antibiotic vancomycin release. J Mater Sci Mater Med. 2005;16(3):189-195.
17. Zhu Y, Wu C, Ramaswamy Y, et al. Preparation, characterization and in vitro bioactivity of mesoporous bioactive glasses (MBGs) scaffolds for bone tissue engineering. Microporous Mesoporous Mater. 2008; 112(1-3):494-503.

18. Kilian KA, Bocking T, Gaus K, King-Lacroix J, Gal M, Gooding JJ. Hybrid lipid bilayers in nanostructured silicon: a biomimetic mesoporous scaffold for optical detection of cholera toxin. Chem Commun (Camb). 2007;(19):1936-1938.

19. Rezwan K, Chen QZ, Blaker JJ, Boccaccini AR. Biodegradable and bioactive porous polymer/inorganic composite scaffolds for bone tissue engineering. Biomaterials. 2006;27(18):3413-3431.

20. Wei J, Chen F, Shin JW, et al. Preparation and characterization of bioactive mesoporous wollastonite-polycaprolactone composite scaffold. Biomaterials. 2009;30(6):1080-1088.

21. Yun HS, Kim SE, Hyun YT, Heo SJ, Shin JW. Three-dimensional mesoporous-giantporous inorganic/organic composite scaffolds for tissue engineering. Chem Mater. 2007;19(26):6363-6366.

22. Wu C, Ramaswamy Y, Zreiqat $\mathrm{H}$. Porous diopside $\left(\mathrm{CaMgSi}_{2} \mathrm{O}_{6}\right)$ scaffold: a promising bioactive material for bone tissue engineering. Acta Biomater. 2010;6:2237-2245.

23. Leonor IB, Ito A, Onuma K, Kanzaki N, Reis RL. In vitro bioactivity of starch thermoplastic/hydroxyapatite composite biomaterials: an in situ study using atomic force microscopy. Biomaterials. 2003;24(4): $579-585$.

24. Li C, Jin HJ, Botsaris GD, Kaplan DL. Silk apatite composites from electrospun fibers. J Mater Res. 2005;20(12):3374-3384.

25. Cool SM, Kenny B, Wu A, et al. Poly(3-hydroxybutyrate-co-3-hydroxyvalerate) composite biomaterials for bone tissue regeneration: in vitro performance assessed by osteoblast proliferation, osteoclast adhesion and resorption, and macrophage proinflammatory response. J Biomed Mater Res A. 2007;82(3):599-610.

26. Xue W, Liu X, Zheng X, Ding C. In vivo evaluation of plasma-sprayed wollastonite coating. Biomaterials. 2005;26(17):3455-3460.

27. Kobayashi T, Okada K, Kuroda T, Sato K. Osteogenic cell cytotoxicity and biomechanical strength of the new ceramic Diopside. J Biomed Mater Res. 1997;37(1):100-107.

28. Taylor MS, Daniels AU, Andriano KP, Heller J. Six bioabsorbable polymers: in vitro acute toxicity of accumulated degradation products. J Appl Biomater. 1994;5(2):151-157.

29. Xynos ID, Hukkanen MV, Batten JJ, Buttery LD, Hench LL, Polak JM Bioglass 45S5 stimulates osteoblast turnover and enhances bone formation in vitro: implications and applications for bone tissue engineering. Calcif Tissue Int. 2000;67(4):321-329.

30. Wei M, Evans JH, Bostrom T, Grondahl L. Synthesis and characterization of hydroxyapatite, fluoride-substituted hydroxyapatite and fluorapatite. J Mater Sci Mater Med. 2003;14(4):311-320.
International Journal of Nanomedicine

\section{Publish your work in this journal}

The International Journal of Nanomedicine is an international, peerreviewed journal focusing on the application of nanotechnology in diagnostics, therapeutics, and drug delivery systems throughout the biomedical field. This journal is indexed on PubMed Central, MedLine, CAS, SciSearch ${ }^{\circledR}$, Current Contents ${ }^{\circledR} /$ Clinical Medicine,

\section{Dovepress}

Journal Citation Reports/Science Edition, EMBase, Scopus and the Elsevier Bibliographic databases. The manuscript management system is completely online and includes a very quick and fair peer-review system, which is all easy to use. Visit http://www.dovepress.com/ testimonials.php to read real quotes from published authors. 